\title{
Regionalización de la cirugía oncológica torácica en Chile
}

\author{
Regionalization of thoracic oncology surgery in Chile
}

Pablo Pérez C. .,2, Raimundo Santolaya C. ${ }^{1,3}$, Raúl Berríos S. ${ }^{1,4}$, José Ortega S. ${ }^{1,5}$ y Manfred Zink R. ${ }^{1,6}$

El cáncer representa la segunda causa de muerte en Chile desde hace al menos 20 años $^{1}$ y ha mantenido una tasa de incidencia estable que fluctúa alrededor de los 151 x 100.000 habitantes/año ${ }^{2}$. Existe literatura nacional entre el 2000 y el 2010 que muestra importantes diferencias en las tasas de mortalidad por cáncer según nivel educacional ${ }^{3}$, aun cuando a la fecha no existen datos nacionales sobre diferencias en resultados clínicos entre centros de alto y bajo volumen.

Si bien se han discutido y aprobado importantes políticas públicas como la protección financiera para diagnósticos y tratamientos de alto costo, nombrada en honor de Ricarte Soto ${ }^{4,5}$, la ley del cáncer, que lleva el nombre del póstumo cirujano nacional Dr. Claudio Mora $^{6,7}$ y de la existencia de 17 patologías oncológicas de alta frecuencia garantizadas por el Sistema de Garantía Explícitas en Salud ${ }^{8}$, basta haber trabajado en un hospital público para evidenciar las dificultades sistémicas que existen para proporcionar un cuidado oncológico de alta calidad a nuestra población más vulnerable.

A pesar de que suene paradójico, la cirugía oncológica no se optimiza necesariamente con más cirujanos. Es un problema más complejo que eso.

Cinco ejemplos donde no basta sólo con el cirujano:

1. Un(a) paciente con un nódulo pulmonar periférico y medicamente inoperable: Este paciente necesitaría una biopsia percutánea realizada por un radiólogo con experiencia.

2. Un(a) paciente con un gran tumor central que presenta hemoptisis masiva: Este paciente necesitaría idealmente una angioembolización de las arterias bronquiales realizada por un radiólogo intervencional.

3. Un(a) paciente con un derrame pleural maligno que no reexpande y un riesgo operatorio elevado: este paciente necesitaría un buen citopatólogo para hacer el diagnóstico y un catéter pleural permanente para paliar sus síntomas.

4. Un(a) paciente con una metástasis pulmonar no palpable: este paciente podría necesitar un radiólogo para marcar el nódulo y luego una cirugía.

5. Un(a) paciente con cáncer de pulmón metastásico: este paciente necesitaría acceso a marcadores moleculares y terapias target $\mathrm{o}$ inmunoterapia.

La lista podría ser más extensa, pero el argumento es el mismo, la lucha contra el cáncer es una pelea que los cirujanos no debemos ni podemos dar solos. Se necesita una discusión oncológica multidisciplinaria mediante un comité establecido.

Una deuda pendiente de la oncología chilena con los usuarios de nuestros sistemas de salud es concentrar el manejo del cáncer en centros regionales predeterminados que cuenten tanto con los recursos materiales, como con la experiencia en el manejo de pacientes oncológicos.

La regionalización del cáncer no es algo nuevo. Bendzak et al. ${ }^{9}$, en Canadá, mostraron que concentrar la cirugía oncológica torácica en 14 hospitales en la provincia de Ontario redujo la mortalidad operatoria de $4,1 \%$ a $2,9 \%$ (OR $0,68,95 \%$ CI $0,58-0,81)$ y la estadía hospitalaria en $7 \%$ por año. Darling ${ }^{10}$, también muestra cómo el aumento de volumen se asocia a una disminución de la mortalidad, directamente asociada a la experiencia.

Si bien Chile cuenta con un sistema mixto de salud (público y privado), la regionalización es algo factible que se podría realizar acorde a zonas geográficas, con un número predeterminado de centros de cáncer determinados por el número de habitantes. La Sociedad de Cirujanos de Chile ha avanzado en la dirección correcta en conjunto con el Ministerio de Salud intentando normar y establecer directrices al respecto.

Para concluir, la patología oncológica representa
'Clínica Alemana de Santiago, Universidad del Desarrollo. Santiago, Chile.

'Hospital San Juan de

Dios, Universidad de Chile. Santiago, Chile. Instituto Nacional del

Tórax, Universidad de Chile. Santiago, Chile.

${ }^{4}$ Hospital FACH, Universidad de Valparaíso. Valparaíso, Chile.

${ }^{5}$ Hospital Padre Hurtado. Santiago, Chile. CClínica Dávila. Santiago, Chile.

Recibido 2021-01-26 y aceptado 2021-01-29

Correspondencia a: Dr. Pablo Perez C. dr.pabloperezc@gmail.com 
la segunda causa de muerte en Chile, si bien se han realizado importantes avances como las Garantías Explícitas en Salud para 17 patologías malignas, la Ley Ricarte Soto para enfermedades de alto costo y la Ley del Cáncer Dr. Claudio Mora, aún existen importantes diferencias de sobrevida basados en estrato socioeconómico. Concentrar centros de cáncer en Chile ayudaría precisamente a optimizar recursos para cuando el paciente no necesita solo un cirujano, sino un equipo completo dedicado a tratar cáncer.

\section{Bibliografía}

1. Medina LE, Kaempffer RA. Mortalidad del adulto en Chile. Rev Med Chile [Internet]. 2000;128:1144-9. Available from: https://scielo.conicyt.cl/scielo. php?script=sci_abstract\&pid=S003498872000001000011\&lng=es\&nrm=iso\&t lng=es [citado el 24 de enero de 2021].

2. Medina LE, Kaempffer RA.

Tendencias y características de la mortalidad chilena 19702003. Rev Med Chile [Internet]. 2007;135:240-50. Available from: https://scielo.conicyt.cl/scielo. php?script=sci_abstract\&pid=S0034$98872007000200014 \& \operatorname{lng}=\mathrm{es} \& n r m=$ iso \& tlng=es [citado el 24 de enero de 2021].

3. Herrera Riquelme CA, Kuhn-Barrientos L, Rosso Astorga R, Jiménez de la Jara J. Tendencia de la mortalidad por cáncer en Chile según diferencias por nivel educacional, 2000-2010. Rev Panam
Salud Publica [Internet]. 2015;37:44-51. Available from: https://www.scielosp.org/ article/rpsp/2015.v37n1/44-51/ [citado el 24 de enero de 2021].

4. Chile B del CN de Ley Fácil [Internet]. Biblioteca del Congreso Nacional de Chile; 2014. Available from: https://www. bcn.cl/leyfacil [citado el 24 de enero de 2021].

5. Ley Ricarte Soto [Internet]. Orientación en Salud. Superintendencia de Salud, Gobierno de Chile. [cited 2021 Jan 24]. Available from: http://www. supersalud.gob.cl/difusion/665/w3propertyvalue-6088.html.

6. Nacional B del C. Biblioteca del Congreso Nacional | Ley Chile [Internet]. www.bcn. $\mathrm{cl} /$ leychile. 2020. Available from: https:// www.bcn.cl/leychile [citado el 24 de enero de 2021].

7. Editorial E. La cruzada eterna del doctor Mora | Colegio Médico de Chile [Internet]. Available from: http://revista. colegiomedico.cl/la-cruzada-eterna-deldoctor-mora/ [citado el 24 de enero de 2021].

8. Garantías Explícitas en Salud (GES) [Internet]. Orientación en Salud. Superintendencia de Salud, Gobierno de Chile. Available from: http://www. supersalud.gob.cl/difusion/665/w3propertyvalue-1962.html [citado el 24 de enero de 2021]

9. Bendzsak AM, Baxter NN, Darling GE, Austin PC, Urbach DR. Regionalization and Outcomes of Lung Cancer Surgery in Ontario, Canada. J Clin Oncol. 2017;35:2772-80.

10. Darling GE. Regionalization in thoracic surgery: The importance of the team. The Journal of Thoracic and Cardiovascular Surgery [Internet]. 2021;161:323-9. Available from: https:// linkinghub.elsevier.com/retrieve/pii/ S0022522320321930 [citado el 24 de enero de 2021] 